Evol utes and focal surfaces of $f r$ aned i mmer si ons i $n$ the Eucl i dean space

\begin{tabular}{|l|l|}
\hline 著者 & HONDA Shuni chi, TAKAHASH Nasat ond \\
\hline j our nal or & $\begin{array}{l}\text { Proceedi ngs of the Royal Soci et y of Edi nburgh. } \\
\text { publ i cat i on t i tl e } \\
\text { Sect. A, Nat hemat i cs }\end{array}$ \\
\hline year & 2019- 01- 26 \\
\hline URL & ht t p: //hdl . handl e. net /10258/00009990 \\
\hline
\end{tabular}




\title{
Evolutes and focal surfaces of framed immersions in the Euclidean space
}

\author{
Shun'ichi Honda \\ Department of Mathematics, Hokkaido University Sapporo 060-0810, \\ Japan (s-honda@math.sci.hokudai.ac.jp) \\ Masatomo Takahashi \\ Muroran Institute of Technology, Muroran 050-8585, Japan \\ (masatomo@mmm.muroran-it.ac.jp) \\ (MS received 'Received date'; 'Accepted date')
}

\begin{abstract}
We consider a smooth curve with singular points in the Euclidean space. As a smooth curve with singular points, we have introduced a framed curve or a framed immersion. A framed immersion is a smooth curve with a moving frame and the pair is an immersion. We define an evolute and a focal surface of a framed immersion in the Euclidean space. The evolutes and focal surfaces of framed immersions are generalizations of each object of regular space curves. We give relationships between singularities of the evolutes and of the focal surfaces. Moreover, we consider properties of the evolutes, focal surfaces and repeated evolutes.
\end{abstract}

Keywords: evolute; focal surface; framed immersion; singular point

2010 Mathematics Subject classification: 58K05; 53A04; 53A05; 57R45

\section{Introduction}

For a Frenet curve (i.e. a regular space curve with the linear independent condition), the evolute and the focal surface are classical objects in differential geometry. There are many articles concerning the evolute (i.e. the locus of the centre of osculating spheres) and the focal surface (i.e. the envelope of normal planes) of a Frenet curve (for instance, $[3,4,8,14,15,16]$ ). The image of the evolute is coincides with the set of singular values of the focal surface under a certain condition. They are investigated by using the Frenet frame. However, the evolute and the focal surface may have singular points, even if the original curve is regular. That is the evolute may not have the Frenet frame. Therefore, we cannot consider not only the curvature and the torsion of the evolute, but also repeated evolutes.

In this paper, we consider the evolute and the focal surface of a space curve with singular points. In order to define them, we apply the theory of framed curves (cf. [10]). A framed curve is a space curve with a moving frame. If a framed curve is an immersion, we call a framed immersion. In $\S 2$, we give the definition of framed curves and basic notations. In $\S 3$, we define the evolute and the focal surface of a framed immersion under a certain condition. They are natural generalizations of each object of a Frenet curve. We investigated basic properties of them. For 
example, the evolute is also a framed immersion, see Proposition 3.4. Moreover, we give the relationships between singular points of the evolute and of the focal surface by using the criterion of singularities of smooth mappings (cf. [13]), see Theorem 3.9. In $\S 4$, we focus the evolute. In particular, we introduce the notion of support functions and parallel curves. The focal surface and the evolute are given by the discriminant and the secondary discriminant sets of the support function, respectively. The evolute and the evolutes of parallel curves coincides. We give the relationship between a framed curve in a sphere and the evolute. Furthermore, we consider contact between framed curves and the evolutes of them. Since the evolute is also a framed immersion, we give the $k$-th evolute of the framed immersion in $\S 4.5$. We give examples to understand the phenomena for evolutes and focal surfaces of framed immersions in $\S 5$.

All maps and manifolds considered here are differential of class $C^{\infty}$.

Acknowledgements. The authors would like to thank the referee for helpful comments to improve the original manuscript. The second author was supported by JSPS KAKENHI Grant Number JP 17K05238.

\section{Basic definitions and notations}

Let $\mathbb{R}^{3}$ be the 3 -dimensional Euclidean space equipped with the inner product $\boldsymbol{a} \cdot \boldsymbol{b}=a_{1} b_{1}+a_{2} b_{2}+a_{3} b_{3}$, where $\boldsymbol{a}=\left(a_{1}, a_{2}, a_{3}\right), \boldsymbol{b}=\left(b_{1}, b_{2}, b_{3}\right) \in \mathbb{R}^{3}$. The norm of $\boldsymbol{a}$ is given by $|\boldsymbol{a}|=\sqrt{\boldsymbol{a} \cdot \boldsymbol{a}}$. We define the vector product of $\boldsymbol{a}$ and $\boldsymbol{b}$ by

$$
\boldsymbol{a} \times \boldsymbol{b}=\left|\begin{array}{ccc}
\boldsymbol{e}_{1} & \boldsymbol{e}_{2} & \boldsymbol{e}_{3} \\
a_{1} & a_{2} & a_{3} \\
b_{1} & b_{2} & b_{3}
\end{array}\right|
$$

where $\left\{\boldsymbol{e}_{1}, \boldsymbol{e}_{2}, \boldsymbol{e}_{3}\right\}$ is the canonical basis on $\mathbb{R}^{3}$. We define a set

$$
\Delta=\left\{\boldsymbol{\nu}=\left(\nu_{1}, \nu_{2}\right) \in S^{2} \times S^{2} \mid \nu_{1} \cdot \nu_{2}=0\right\},
$$

where $S^{2}=\left\{\boldsymbol{x} \in \mathbb{R}^{3}|| \boldsymbol{x} \mid=1\right\}$. Then $\Delta$ is a 3 -dimensional smooth manifold.

We quickly review on the evolute and the focal surface of a Frenet curve. Let $I \subset \mathbb{R}$ be an interval. Suppose that $\gamma: I \rightarrow \mathbb{R}^{3}$ is a Frenet curve, that is, $\dot{\gamma}(t)$ and $\ddot{\gamma}(t)$ are linear independent for all $t \in I$, where $\dot{\gamma}(t)=(d \gamma / d t)(t)$ and $\ddot{\gamma}(t)=$ $\left(d^{2} \gamma / d t^{2}\right)(t)$. Then we have the Frenet frame $\{\boldsymbol{t}(t), \boldsymbol{n}(t), \boldsymbol{b}(t)\}$ along $\gamma(t)$, where

$$
\boldsymbol{t}(t)=\frac{\dot{\gamma}(t)}{|\dot{\gamma}(t)|}, \boldsymbol{n}(t)=\frac{(\dot{\gamma}(t) \times \ddot{\gamma}(t)) \times \dot{\gamma}(t)}{|(\dot{\gamma}(t) \times \ddot{\gamma}(t)) \times \dot{\gamma}(t)|}, \boldsymbol{b}(t)=\frac{\dot{\gamma}(t) \times \ddot{\gamma}(t)}{|\dot{\gamma}(t) \times \ddot{\gamma}(t)|}
$$

By the standard arguments, we have the following Frenet-Serret formula:

$$
\left(\begin{array}{c}
\dot{\boldsymbol{t}}(t) \\
\dot{\boldsymbol{n}}(t) \\
\dot{\boldsymbol{b}}(t)
\end{array}\right)=\left(\begin{array}{ccc}
0 & |\dot{\gamma}(t)| \kappa(t) & 0 \\
-|\dot{\gamma}(t)| \kappa(t) & 0 & |\dot{\gamma}(t)| \tau(t) \\
0 & -|\dot{\gamma}(t)| \tau(t) & 0
\end{array}\right)\left(\begin{array}{c}
\boldsymbol{t}(t) \\
\boldsymbol{n}(t) \\
\boldsymbol{b}(t)
\end{array}\right)
$$

where $\kappa(t)=|\dot{\gamma}(t) \times \ddot{\gamma}(t)| /|\dot{\gamma}(t)|^{3}$ and $\tau(t)=\operatorname{det}(\dot{\gamma}(t), \ddot{\gamma}(t), \dddot{\gamma}(t)) /|\dot{\gamma}(t) \times \ddot{\gamma}(t)|^{2}$. We call $\kappa(t)$ the curvature and $\tau(t)$ the torsion of $\gamma(t)$. 
The evolute $E v(\gamma): I \rightarrow \mathbb{R}^{3}$ of a Frenet curve $\gamma$ is given by

$$
E v(\gamma)(t)=\gamma(t)+\frac{1}{\kappa(t)} \boldsymbol{n}(t)-\frac{\dot{\kappa}(t)}{|\dot{\gamma}(t)| \kappa^{2}(t) \tau(t)} \boldsymbol{b}(t),
$$

away from points where $\tau(t)=0$ (cf. $[3,4,8,14,15,16])$. The evolute $E v(\gamma)$ of a Frenet curve is given by the locus of the centre of osculating spheres. A Frenet curve and its osculating sphere have contact of order at least three at the point.

On the other hand, we can not construct the Frenet frame at a singular point or a point which does not satisfy the linear independent condition, so that we can not define the evolute as the above. In this paper, we investigated the evolute of a space curve with singular points in the Euclidean space, see Definition 3.1. It is a natural generalization of the evolute of a Frenet curve.

The focal surface $F S(\gamma): I \times \mathbb{R} \rightarrow \mathbb{R}^{3}$ of a Frenet curve $\gamma$ is given by

$$
F S(\gamma)(t, \lambda)=\gamma(t)+\frac{1}{\kappa(t)} \boldsymbol{n}(t)+\lambda \boldsymbol{b}(t)
$$

If $\tau(t) \neq 0$ for all $t \in I$, then the set of singular values of the focal surface coincides with the image of the evolute. Therefore, there is the important relationship between the evolute and the focal surface.

A framed curve in the Euclidean space is a smooth curve with a moving frame (cf. [10]). It is a generalization of not only a Frenet curve, but also a Legendre curve in the unit tangent bundle over the Euclidean plane (cf. [5]).

Definition 2.1. We say that $\left(\gamma, \nu_{1}, \nu_{2}\right): I \rightarrow \mathbb{R}^{3} \times \Delta$ is a framed curve if $\dot{\gamma}(t)$. $\nu_{1}(t)=0$ and $\dot{\gamma}(t) \cdot \nu_{2}(t)=0$ for all $t \in I$. Moreover, if $\left(\gamma, \nu_{1}, \nu_{2}\right)$ is an immersion, we call $\left(\gamma, \nu_{1}, \nu_{2}\right)$ a framed immersion. Then $\gamma: I \rightarrow \mathbb{R}^{3}$ is called a framed base curve.

Remark 2.2. If $\gamma:\left(I, t_{0}\right) \rightarrow \mathbb{R}^{3}$ is a real analytic curve germ, then $\gamma$ is a framed base curve, that is, there exists a mapping germ $\left(\nu_{1}, \nu_{2}\right):\left(I, t_{0}\right) \rightarrow \Delta$ such that $\left(\gamma, \nu_{1}, \nu_{2}\right)$ is a framed curve, see [10]. See also [7].

We put on $\boldsymbol{\mu}(t)=\nu_{1}(t) \times \nu_{2}(t)$. It follows that $\left\{\nu_{1}(t), \nu_{2}(t), \boldsymbol{\mu}(t)\right\}$ is a moving frame along the framed base curve $\gamma(t)$. By the standard arguments, we have the following Frenet-Serret type formula:

$$
\left(\begin{array}{c}
\dot{\nu_{1}}(t) \\
\dot{\nu_{2}}(t) \\
\dot{\boldsymbol{\mu}}(t)
\end{array}\right)=\left(\begin{array}{ccc}
0 & \ell(t) & m(t) \\
-\ell(t) & 0 & n(t) \\
-m(t) & -n(t) & 0
\end{array}\right)\left(\begin{array}{c}
\nu_{1}(t) \\
\nu_{2}(t) \\
\boldsymbol{\mu}(t)
\end{array}\right), \dot{\gamma}(t)=\alpha(t) \boldsymbol{\mu}(t),
$$

where $\ell(t)=\dot{\nu_{1}}(t) \cdot \nu_{2}(t), m(t)=\dot{\nu_{1}}(t) \cdot \boldsymbol{\mu}(t), n(t)=\dot{\nu_{2}}(t) \cdot \boldsymbol{\mu}(t)$ and $\alpha(t)=\dot{\gamma}(t) \cdot \boldsymbol{\mu}(t)$. We call the mapping $(\ell, m, n, \alpha): I \rightarrow \mathbb{R}^{4}$ the curvature of the framed curve.

Note that $t_{0}$ is a singular point of $\gamma$ if and only if $\alpha\left(t_{0}\right)=0$, and $t_{0}$ is a singular point of $\left(\gamma, \nu_{1}, \nu_{2}\right)$ if and only if $(\ell, m, n, \alpha)\left(t_{0}\right)=\mathbf{0}$. Moreover, we introduce a smooth function $f(t)=\ell(t)\left(m^{2}(t)+n^{2}(t)\right)+m(t) \dot{n}(t)-\dot{m}(t) n(t)$.

Definition 2.3. Let $\left(\gamma, \nu_{1}, \nu_{2}\right)$ and $\left(\widetilde{\gamma}, \widetilde{\nu}_{1}, \widetilde{\nu}_{2}\right): I \rightarrow \mathbb{R}^{3} \times \Delta$ be framed curves. We say that $\left(\gamma, \nu_{1}, \nu_{2}\right)$ and $\left(\widetilde{\gamma}, \widetilde{\nu}_{1}, \widetilde{\nu}_{2}\right)$ are congruent as framed curves if there exist a rotation $A \in S O(3)$ and a translation $\boldsymbol{a} \in \mathbb{R}^{3}$ such that $\widetilde{\gamma}(t)=A(\gamma(t))+\boldsymbol{a}$, $\widetilde{\nu_{1}}(t)=A\left(\nu_{1}(t)\right)$ and $\widetilde{\nu_{2}}(t)=A\left(\nu_{2}(t)\right)$ for all $t \in I$. 
We have the existence and the uniqueness theorems for framed curves similarly to the case of Frenet curves.

Theorem 2.4. (The Existence Theorem, [10]) Let $(\ell, m, n, \alpha): I \rightarrow \mathbb{R}^{4}$ be a smooth mapping. There exists a framed curve $\left(\gamma, \nu_{1}, \nu_{2}\right): I \rightarrow \mathbb{R}^{3} \times \Delta$ whose curvature is $(\ell, m, n, \alpha)$.

Theorem 2.5. (The Uniqueness Theorem, [10]) Let $\left(\gamma, \nu_{1}, \nu_{2}\right)$ and $\left(\widetilde{\gamma}, \widetilde{\nu}_{1}, \widetilde{\nu}_{2}\right)$ : $I \rightarrow \mathbb{R}^{3} \times \Delta$ be framed curves with the curvature $(\ell, m, n, \alpha)$ and $(\tilde{\ell}, \widetilde{m}, \widetilde{n}, \widetilde{\alpha})$, respectively. Then $\left(\gamma, \nu_{1}, \nu_{2}\right)$ and $\left(\widetilde{\gamma}, \widetilde{\nu}_{1}, \widetilde{\nu}_{2}\right)$ are congruent as framed curves if and only if $(\ell, m, n, \alpha)=(\widetilde{\ell}, \widetilde{m}, \widetilde{n}, \widetilde{\alpha})$.

Let $I$ and $\bar{I}$ be intervals. A smooth function $s: \bar{I} \rightarrow I$ is a change of parameter when $s$ is surjective and has a positive derivative at every point. It follows that $s$ is a diffeomorphism by calculus. Let $\left(\gamma, \nu_{1}, \nu_{2}\right): I \rightarrow \mathbb{R}^{3} \times \Delta$ and $\left(\bar{\gamma}, \bar{\nu}_{1}, \bar{\nu}_{2}\right)$ : $\bar{I} \rightarrow \mathbb{R}^{3} \times \Delta$ be framed curves with the curvatures $(\ell, m, n, \alpha)$ and $(\bar{\ell}, \bar{m}, \bar{n}, \bar{\alpha})$, respectively. Suppose that $\left(\gamma, \nu_{1}, \nu_{2}\right)$ and $\left(\bar{\gamma}, \bar{\nu}_{1}, \bar{\nu}_{2}\right)$ are parametrically equivalent via the change of parameter $s: \bar{I} \rightarrow I$, that is, $\left(\bar{\gamma}, \bar{\nu}_{1}, \bar{\nu}_{2}\right)(t)=\left(\gamma, \nu_{1}, \nu_{2}\right)(s(t))$ for all $t \in \bar{I}$. By differentiation, we have

$$
(\bar{\ell}(t), \bar{m}(t), \bar{n}(t), \bar{\alpha}(t))=\dot{s}(t)(\ell(s(t)), m(s(t)), n(s(t)), \alpha(s(t))) .
$$

Therefore, the curvature of a framed curve is depend on a parametrization.

For the normal planes of $\gamma(t)$, spanned by $\nu_{1}(t)$ and $\nu_{2}(t)$, there are other frames by rotations and reflections. We define $\left(\bar{\nu}_{1}(t), \bar{\nu}_{2}(t)\right) \in \Delta$ by

$$
\left(\begin{array}{l}
\bar{\nu}_{1}(t) \\
\bar{\nu}_{2}(t)
\end{array}\right)=\left(\begin{array}{cc}
\cos \theta(t) & -\sin \theta(t) \\
\sin \theta(t) & \cos \theta(t)
\end{array}\right)\left(\begin{array}{c}
\nu_{1}(t) \\
\nu_{2}(t)
\end{array}\right)
$$

where $\theta(t)$ is a smooth function. Then $\left(\gamma, \bar{\nu}_{1}, \bar{\nu}_{2}\right): I \rightarrow \mathbb{R}^{3} \times \Delta$ is a framed curve and $\overline{\boldsymbol{\mu}}(t)=\bar{\nu}_{1}(t) \times \bar{\nu}_{2}(t)=\nu_{1}(t) \times \nu_{2}(t)=\boldsymbol{\mu}(t)$. The curvature $(\bar{\ell}(t), \bar{m}(t), \bar{n}(t), \bar{\alpha}(t))$ of $\left(\gamma, \bar{\nu}_{1}, \bar{\nu}_{2}\right)$ is given by

$$
(\ell(t)-\dot{\theta}(t), m(t) \cos \theta(t)-n(t) \sin \theta(t), m(t) \sin \theta(t)+n(t) \cos \theta(t), \alpha(t)) .
$$

We call the moving frame $\left\{\bar{\nu}_{1}(t), \bar{\nu}_{2}(t), \boldsymbol{\mu}(t)\right\}$ a rotated frame along $\gamma(t)$ by $\theta(t)$. If we take a smooth function $\theta: I \rightarrow \mathbb{R}$ which satisfies $\dot{\theta}(t)=\ell(t)$, then we call the rotated frame $\left\{\bar{\nu}_{1}(t), \bar{\nu}_{2}(t), \boldsymbol{\mu}(t)\right\}$ an adapted frame along $\gamma(t)$ by $\theta(t)$ (cf. $\left.[2,10]\right)$.

On the other hand, we define $\left(\widetilde{\nu}_{1}(t), \widetilde{\nu}_{2}(t)\right) \in \Delta$ by

$$
\left(\begin{array}{c}
\widetilde{\nu}_{1}(t) \\
\widetilde{\nu}_{2}(t)
\end{array}\right)=\left(\begin{array}{cc}
1 & 0 \\
0 & -1
\end{array}\right)\left(\begin{array}{cc}
\cos \theta(t) & -\sin \theta(t) \\
\sin \theta(t) & \cos \theta(t)
\end{array}\right)\left(\begin{array}{l}
\nu_{1}(t) \\
\nu_{2}(t)
\end{array}\right),
$$

where $\theta(t)$ is a smooth function. Then $\left(\gamma, \widetilde{\nu}_{1}, \widetilde{\nu}_{2}\right): I \rightarrow \mathbb{R}^{3} \times \Delta$ is a framed curve and $\widetilde{\boldsymbol{\mu}}(t)=\widetilde{\nu}_{1}(t) \times \widetilde{\nu}_{2}(t)=\nu_{2}(t) \times \nu_{1}(t)=-\boldsymbol{\mu}(t)$. The curvature $(\widetilde{\ell}(t), \widetilde{m}(t), \widetilde{n}(t), \widetilde{\alpha}(t))$ of $\left(\gamma, \widetilde{\nu}_{1}, \widetilde{\nu}_{2}\right)$ is given by

$$
(-\ell(t)+\dot{\theta}(t),-m(t) \cos \theta(t)+n(t) \sin \theta(t), m(t) \sin \theta(t)+n(t) \cos \theta(t),-\alpha(t)) .
$$

We call the moving frame $\left\{\widetilde{\nu}_{1}(t), \widetilde{\nu}_{2}(t),-\boldsymbol{\mu}(t)\right\}$ a reflected frame along $\gamma(t)$ by $\theta(t)$.

By a direct calculation, we have the following result. 
Proposition 2.6. Under the above notations, we have the following:

(1) $m^{2}(t)+n^{2}(t)=\bar{m}^{2}(t)+\bar{n}^{2}(t)=\widetilde{m}^{2}(t)+\widetilde{n}^{2}(t)$

(2) $f(t)=\bar{f}(t)=-\tilde{f}(t)$.

We now consider a special moving frame along a framed base curve under a condition. Let $\left(\gamma, \nu_{1}, \nu_{2}\right): I \rightarrow \mathbb{R}^{3} \times \Delta$ be a framed curve with $m^{2}(t)+n^{2}(t) \neq 0$. Then we define $\left(\boldsymbol{n}_{1}(t), \boldsymbol{n}_{2}(t)\right) \in \Delta$ by

$$
\boldsymbol{n}_{1}(t)=\frac{m(t) \nu_{1}(t)+n(t) \nu_{2}(t)}{\sqrt{m^{2}(t)+n^{2}(t)}}, \boldsymbol{n}_{2}(t)=\frac{-n(t) \nu_{1}(t)+m(t) \nu_{2}(t)}{\sqrt{m^{2}(t)+n^{2}(t)}} .
$$

By a direct calculation, $\left(\gamma, \boldsymbol{n}_{1}, \boldsymbol{n}_{2}\right): I \rightarrow \mathbb{R}^{3} \times \Delta$ is a framed immersion and $\boldsymbol{n}_{1}(t) \times \boldsymbol{n}_{2}(t)=\boldsymbol{\mu}(t)$. We call the moving frame $\left\{\boldsymbol{n}_{1}(t), \boldsymbol{n}_{2}(t), \boldsymbol{\mu}(t)\right\}$ a Frenet type frame along $\gamma(t)$. Then the Frenet-Serret type formula is given by

$$
\left(\begin{array}{c}
\dot{\boldsymbol{n}}_{1}(t) \\
\dot{\boldsymbol{n}}_{2}(t) \\
\dot{\boldsymbol{\mu}}(t)
\end{array}\right)=\left(\begin{array}{ccc}
0 & L(t) & M(t) \\
-L(t) & 0 & 0 \\
-M(t) & 0 & 0
\end{array}\right)\left(\begin{array}{c}
\boldsymbol{n}_{1}(t) \\
\boldsymbol{n}_{2}(t) \\
\boldsymbol{\mu}(t)
\end{array}\right), \dot{\gamma}(t)=\alpha(t) \boldsymbol{\mu}(t),
$$

where

$$
L(t)=f(t) /\left(m^{2}(t)+n^{2}(t)\right), M(t)=\sqrt{m^{2}(t)+n^{2}(t)} .
$$

Therefore, the curvature of the framed immersion $\left(\gamma, \boldsymbol{n}_{1}, \boldsymbol{n}_{2}\right)$ is given by $(L, M, 0, \alpha)$.

Since the original frame $\left\{\nu_{1}(t), \nu_{2}(t), \boldsymbol{\mu}(t)\right\}$ and the Frenet type frame $\left\{\boldsymbol{n}_{1}(t)\right.$, $\left.\boldsymbol{n}_{2}(t), \boldsymbol{\mu}(t)\right\}$ have the common unit vector $\boldsymbol{\mu}(t)$ and the same orientation, the Frenet type frame is one of a rotated frame along $\gamma(t)$.

Let $\gamma: I \rightarrow \mathbb{R}^{3}$ be a Frenet curve. If we take $\nu_{1}(t)=\boldsymbol{n}(t)$ and $\nu_{2}(t)=\boldsymbol{b}(t)$, then $\left(\gamma, \nu_{1}, \nu_{2}\right): I \rightarrow \mathbb{R}^{3} \times \Delta$ is a framed curve and we have $\boldsymbol{n}_{1}(t)=-\boldsymbol{n}(t), \boldsymbol{n}_{2}(t)=$ $-\boldsymbol{b}(t), \boldsymbol{\mu}(t)=\boldsymbol{t}(t)$. This is the reason why we call $\left\{\boldsymbol{n}_{1}(t), \boldsymbol{n}_{2}(t), \boldsymbol{\mu}(t)\right\}$ the Frenet type frame along $\gamma(t)$.

\section{Evolutes and focal surfaces of framed immersions}

In this section we investigate the evolute and the focal surface of a framed immersion. Let $\left(\gamma, \nu_{1}, \nu_{2}\right): I \rightarrow \mathbb{R}^{3} \times \Delta$ be a framed immersion with $f(t) \neq 0$.

Definition 3.1. The evolute $\mathcal{E} v(\gamma): I \rightarrow \mathbb{R}^{3}$ of the framed immersion $\left(\gamma, \nu_{1}, \nu_{2}\right)$ is defined by

$$
\begin{aligned}
\mathcal{E} v(\gamma)(t)=\gamma(t) & -\frac{\alpha(t) \dot{n}(t)-\dot{\alpha}(t) n(t)+\alpha(t) \ell(t) m(t)}{f(t)} \nu_{1}(t) \\
& +\frac{\alpha(t) \dot{m}(t)-\dot{\alpha}(t) m(t)-\alpha(t) \ell(t) n(t)}{f(t)} \nu_{2}(t) .
\end{aligned}
$$

For a Frenet curve $\gamma: I \rightarrow \mathbb{R}^{3}$ with $\tau(t) \neq 0$, if we take $\nu_{1}(t)=\boldsymbol{n}(t)$ and $\nu_{2}(t)=$ $\boldsymbol{b}(t)$, then $\left(\gamma, \nu_{1}, \nu_{2}\right)$ is a framed immersion with $f(t) \neq 0$. By a direct calculation, we have $\mathcal{E} v(\gamma)(t)=E v(\gamma)(t)$. Hence, Definition 3.1 is a natural generalization of the definition of the evolute of a Frenet curve. Moreover, $\left(E_{v}(\gamma), \boldsymbol{t}, \boldsymbol{n}\right)$ is a framed immersion. 
Remark 3.2. Let $s: \bar{I} \rightarrow I$ be a change of parameter, where $I$ and $\bar{I}$ are intervals. Let $\left(\gamma, \nu_{1}, \nu_{2}\right): I \rightarrow \mathbb{R}^{3} \times \Delta$ and $\left(\bar{\gamma}, \bar{\nu}_{1}, \bar{\nu}_{2}\right): \bar{I} \rightarrow \mathbb{R}^{3} \times \Delta$ be framed immersions with $f(t) \neq 0$. Suppose that $\left(\gamma, \nu_{1}, \nu_{2}\right)$ and $\left(\bar{\gamma}, \bar{\nu}_{1}, \bar{\nu}_{2}\right)$ are parametrically equivalent via the change of parameter $s$, that is, $\left(\bar{\gamma}, \bar{\nu}_{1}, \bar{\nu}_{2}\right)(t)=\left(\gamma, \nu_{1}, \nu_{2}\right)(s(t))$ for all $t \in I$. By $(2.1)$, we have $\bar{f}(t)=(\dot{s}(t))^{3} f(s(t)) \neq 0$ and $\mathcal{E} v(\bar{\gamma})(t)=\mathcal{E} v(\gamma)(s(t))$. In this sense, the evolute of a framed immersion is independent on the choice of a parametrization.

Remark 3.3. We consider a framed immersion $\left(\gamma, \bar{\nu}_{1}, \bar{\nu}_{2}\right): I \rightarrow \mathbb{R}^{3} \times \Delta$, where $\left\{\bar{\nu}_{1}(t), \bar{\nu}_{2}(t), \boldsymbol{\mu}(t)\right\}$ is a rotated frame along $\gamma(t)$ by $\theta(t)$. Since Proposition 2.6, we have $\bar{f}(t) \neq 0$. By a direct calculation, the evolute of $\left(\gamma, \nu_{1}, \nu_{2}\right)$ coincides with the evolute of $\left(\gamma, \bar{\nu}_{1}, \bar{\nu}_{2}\right)$. Similarly, we can show the evolute of a framed immersion is independent on reflections of the moving frame.

Proposition 3.4. The evolute $\mathcal{E} v(\gamma)$ is a framed base curve. More precisely, $(\mathcal{E} v(\gamma)$, $\left.\boldsymbol{\mu}, \boldsymbol{n}_{1}\right): I \rightarrow \mathbb{R}^{3} \times \Delta$ is a framed immersion with the curvature $\left(-M, 0, L, \alpha_{\mathcal{E} v}\right)$, where

$$
\begin{aligned}
\boldsymbol{n}_{1}= & \frac{1}{\sqrt{m^{2}+n^{2}}}\left(m \nu_{1}+n \nu_{2}\right), M=\sqrt{m^{2}+n^{2}}, L=\frac{f}{m^{2}+n^{2}}, \\
\alpha_{\mathcal{E} v}= & \frac{1}{\sqrt{m^{2}+n^{2}}}\left(n\left(\frac{d}{d t}\left(\frac{\alpha \dot{n}-\dot{\alpha} n+\alpha \ell m}{f}\right)+\ell\left(\frac{\alpha \dot{m}-\dot{\alpha} m-\alpha \ell n}{f}\right)\right)\right. \\
& \left.+m\left(\frac{d}{d t}\left(\frac{\alpha \dot{m}-\dot{\alpha} m-\alpha \ell n}{f}\right)-\ell\left(\frac{\alpha \dot{n}-\dot{\alpha} n+\alpha \ell m}{f}\right)\right)\right) .
\end{aligned}
$$

Proof. By using the Frenet-Serret type formula, we have

$$
\begin{aligned}
\dot{\mathcal{E} v}(\gamma)= & \left(-\frac{d}{d t}\left(\frac{\alpha \dot{n}-\dot{\alpha} n+\alpha \ell m}{f}\right)-\left(\frac{\alpha \dot{m}-\dot{\alpha} m-\alpha \ell n}{f}\right) \ell\right) \nu_{1} \\
& +\left(\frac{d}{d t}\left(\frac{\alpha \dot{m}-\dot{\alpha} m-\alpha \ell n}{f}\right)-\left(\frac{\alpha \dot{n}-\dot{\alpha} n+\alpha \ell m}{f}\right) \ell\right) \nu_{2} .
\end{aligned}
$$

It follows that $\dot{\mathcal{E} v}(\gamma)(t) \cdot \boldsymbol{\mu}(t)=0$ and $\dot{\mathcal{E} v}(\gamma)(t) \cdot \boldsymbol{n}_{1}(t)=0$. Since $\boldsymbol{\mu}(t) \cdot \boldsymbol{n}_{1}(t)=0$ for all $t \in I,\left(\mathcal{E} v(\gamma), \boldsymbol{\mu}, \boldsymbol{n}_{1}\right): I \rightarrow \mathbb{R}^{3} \times \Delta$ is a framed curve. Then we have a moving frame $\left\{\boldsymbol{\mu}(t), \boldsymbol{n}_{1}(t), \boldsymbol{n}_{2}(t)\right\}$ along $\mathcal{E} v(\gamma)(t)$, where $\boldsymbol{n}_{2}(t)=\boldsymbol{\mu}(t) \times \boldsymbol{n}_{1}(t)$. Since $\dot{\mathcal{E} v}(\gamma)(t)=\alpha_{\mathcal{E} v}(t) \boldsymbol{n}_{2}(t)$, the curvature of the framed curve $\left(\mathcal{E} v(\gamma), \boldsymbol{\mu}, \boldsymbol{n}_{1}\right)$ is given by $\left(-M(t), 0, L(t), \alpha_{\mathcal{E} v}(t)\right)$. By the assumption, we have $M(t) \neq 0$ and $L(t) \neq 0$. Therefore $\left(\mathcal{E} v(\gamma), \boldsymbol{\mu}, \boldsymbol{n}_{1}\right)$ is a framed immersion.

By Proposition 3.4, $t_{0}$ is a singular point of the evolute $\mathcal{E} v(\gamma)$ if and only if $\alpha_{\mathcal{E} v}\left(t_{0}\right)=0$. Moreover, since $f_{\mathcal{E} v}(t)=-L^{2}(t) M(t) \neq 0$, the framed immersion $\left(\mathcal{E} v(\gamma), \boldsymbol{\mu}, \boldsymbol{n}_{1}\right): I \rightarrow \mathbb{R}^{3} \times \Delta$ has the evolute $\mathcal{E} v(\mathcal{E} v(\gamma))$, see $\S 4.5$.

On the other hand, for a framed immersion $\left(\gamma, \nu_{1}, \nu_{2}\right): I \rightarrow \mathbb{R}^{3} \times \Delta$ with $m^{2}(t)+$ $n^{2}(t) \neq 0$, we define a focal surface.

Definition 3.5. The focal surface $\mathcal{F S}(\gamma): I \times J \rightarrow \mathbb{R}^{3}$ of the framed immersion $\left(\gamma, \nu_{1}, \nu_{2}\right)$ is defined by

$$
\mathcal{F S}(\gamma)(t, a, b)=\gamma(t)-a \nu_{1}(t)-b \nu_{2}(t),
$$

where $J=\left\{(a, b) \in \mathbb{R}^{2} \mid \alpha(t)-a m(t)-b n(t)=0\right.$ for all $\left.t \in I\right\}$. 
At least locally, we may assume $m(t) \neq 0$ or $n(t) \neq 0$. If $m(t) \neq 0$ (respectively, $n(t) \neq 0$ ), then the focal surface $\mathcal{F S}(\gamma): I \times \mathbb{R} \rightarrow \mathbb{R}^{3}$ is given by

$$
\mathcal{F S}(\gamma)(t, b)=\gamma(t)-\left(\frac{\alpha(t)}{m(t)}-b \frac{n(t)}{m(t)}\right) \nu_{1}(t)-b \nu_{2}(t)
$$

$\left(\right.$ respectively, $\left.\mathcal{F} \mathcal{S}(\gamma)(t, a)=\gamma(t)-a \nu_{1}(t)-\left(\frac{\alpha(t)}{n(t)}-a \frac{m(t)}{n(t)}\right) \nu_{2}(t)\right)$.

Remark 3.6. Let $\gamma: I \rightarrow \mathbb{R}^{3}$ be a Frenet curve. If we take $\nu_{1}(t)=\boldsymbol{n}(t)$ and $\nu_{2}(t)=\boldsymbol{b}(t)$, then $\left(\gamma, \nu_{1}, \nu_{2}\right)$ is a framed immersion. Since $m(t)=-|\dot{\gamma}(t)| \kappa(t)$, $n(t)=0$ and $\alpha(t)=|\dot{\gamma}(t)|$, the focal surface $\mathcal{F} \mathcal{S}(\gamma): I \times J \rightarrow \mathbb{R}^{3}$ is given by $\mathcal{F} \mathcal{S}(\gamma)(t, a, b)=\gamma(t)-a \boldsymbol{n}(t)-b \boldsymbol{b}(t)$. Here $J=\left\{(a, b) \in \mathbb{R}^{2} \mid a=-1 / \kappa(t)\right.$ for all $t \in I\}$. Thus $\mathcal{F S}(\gamma)(t, a, b)$ coincides with $F S(\gamma)(t, \lambda)$. In this sense, Definition 3.5 is a natural generalization of the definition of the focal surface of a Frenet curve.

Remark 3.7. Let $\left(\gamma, \nu_{1}, \nu_{2}\right): I \rightarrow \mathbb{R}^{3} \times \Delta$ and $\left(\bar{\gamma}, \bar{\nu}_{1}, \bar{\nu}_{2}\right): \bar{I} \rightarrow \mathbb{R}^{3} \times \Delta$ be framed immersions with $m^{2}(t)+n^{2}(t) \neq 0$. Suppose that $\left(\gamma, \nu_{1}, \nu_{2}\right)$ and $\left(\bar{\gamma}, \bar{\nu}_{1}, \bar{\nu}_{2}\right)$ are parametrically equivalent via the change of parameter $s: \bar{I} \rightarrow I$, that is, $\left(\bar{\gamma}, \bar{\nu}_{1}, \bar{\nu}_{2}\right)(t)=\left(\gamma, \nu_{1}, \nu_{2}\right)(s(t))$ for all $t \in I$. Since $\bar{m}^{2}(t)+\bar{n}^{2}(t) \neq 0$ and

$$
\begin{aligned}
\bar{J} & =\left\{(a, b) \in \mathbb{R}^{2} \mid \bar{\alpha}(t)-a \bar{m}(t)-b \bar{n}(t)=0 \text { for all } t \in \bar{I}\right\} \\
& =\left\{(a, b) \in \mathbb{R}^{2} \mid \alpha(s(t))-a m(s(t))-b n(s(t))=0 \text { for all } t \in \bar{I}\right\},
\end{aligned}
$$

we have $\mathcal{F} \mathcal{S}(\bar{\gamma})(t, a, b)=\mathcal{F} \mathcal{S}(\gamma)(s(t), a, b)$. In this sense, the focal surface of a framed immersion is independent on the choice of a parametrization.

Remark 3.8. The focal surface of $\left(\gamma, \nu_{1}, \nu_{2}\right)$ is $\mathcal{R}$-equivalent to the focal surface of $\left(\gamma, \bar{\nu}_{1}, \bar{\nu}_{2}\right)$, where $\left\{\bar{\nu}_{1}(t), \bar{\nu}_{2}(t), \boldsymbol{\mu}(t)\right\}$ is a rotated frame along $\gamma(t)$ by $\theta(t)$. In fact, if we take a diffeomorphism $\phi: I \times \bar{J} \rightarrow I \times J$ defined by

$$
(t, c, d) \mapsto(t, c \cos \theta(t)+d \sin \theta(t),-c \sin \theta(t)+d \cos \theta(t)),
$$

then $\mathcal{F S}(\gamma)(\phi(t, c, d))=\overline{\mathcal{F S}}(\gamma)(t, c, d)$. Here $\bar{J}=\left\{(c, d) \in \mathbb{R}^{2} \mid \alpha(t)-c \bar{m}(t)-\right.$ $d \bar{n}(t)=0$ for all $t \in I\}$ and $\overline{\mathcal{F} S}(\gamma)(t, c, d): I \times \bar{J} \rightarrow \mathbb{R}^{3}, \overline{\mathcal{F} S}(\gamma)(t, c, d)=\gamma(t)-$ $c \bar{\nu}_{1}(t)-d \bar{\nu}_{2}(t)$. Similarly, the focal surface of $\left(\gamma, \nu_{1}, \nu_{2}\right)$ is $\mathcal{R}$-equivalent to the focal surface of $\left(\gamma, \widetilde{\nu}_{1}, \widetilde{\nu}_{2}\right)$, where $\left\{\widetilde{\nu}_{1}(t), \widetilde{\nu}_{2}(t),-\boldsymbol{\mu}(t)\right\}$ is a reflected frame along $\gamma(t)$ by $\theta(t)$.

For simplicity, we consider a framed immersion by the Frenet type frame. Let $\left(\gamma, \boldsymbol{n}_{1}, \boldsymbol{n}_{2}\right): I \rightarrow \mathbb{R}^{3} \times \Delta$ be a framed immersion with the Frenet type frame $\left\{\boldsymbol{n}_{1}(t), \boldsymbol{n}_{2}(t), \boldsymbol{\mu}(t)\right\}$ and $f(t)=L(t) M^{2}(t) \neq 0$. By Definitions 3.1 and 3.5, we have $\mathcal{E} v(\gamma): I \rightarrow \mathbb{R}^{3}$

$$
\mathcal{E} v(\gamma)(t)=\gamma(t)-\frac{\alpha(t)}{M(t)} \boldsymbol{n}_{1}(t)+\frac{\alpha(t) \dot{M}(t)-\dot{\alpha}(t) M(t)}{L(t) M^{2}(t)} \boldsymbol{n}_{2}(t)
$$

and $\mathcal{F S}(\gamma): I \times \mathbb{R} \rightarrow \mathbb{R}^{3}$,

$$
\mathcal{F S}(\gamma)(t, \lambda)=\gamma(t)-\frac{\alpha(t)}{M(t)} \boldsymbol{n}_{1}(t)-\lambda \boldsymbol{n}_{2}(t)
$$


We show that the set of singular values of the focal surface coincides with the image of the evolute. Moreover, we give relationships between singularities of the evolute and of the focal surface.

Theorem 3.9. Let $\left(\gamma, \nu_{1}, \nu_{2}\right): I \rightarrow \mathbb{R}^{3} \times \Delta$ be a framed immersion with $f(t) \neq 0$. Then we have the following:

(1) The set of singular values of the focal surface $\mathcal{F S}(\gamma)$ coincides with the image of the evolute $\mathcal{E} v(\gamma)$,

(2) The focal surface $\mathcal{F S}(\gamma)$ is locally diffeomorphic to the cuspidal edge $C E$ at $\left(t_{0}, a_{0}, b_{0}\right)$ if and only if $t_{0}$ is a regular point of $\mathcal{E} v(\gamma)$,

(3) The focal surface $\mathcal{F S}(\gamma)$ is locally diffeomorphic to the swallowtail $S W$ at $\left(t_{0}, a_{0}, b_{0}\right)$ if and only if the evolute $\mathcal{E} v(\gamma)$ is locally diffeomorphic to the $(2,3,4)$ type $C$ at $t_{0}$.

Here, $C E:\left(\mathbb{R}^{2}, 0\right) \rightarrow\left(\mathbb{R}^{3}, 0\right),(u, v) \mapsto\left(u, v^{2}, v^{3}\right)$ is the cuspidal edge (Figure 1), $S W:\left(\mathbb{R}^{2}, 0\right) \rightarrow\left(\mathbb{R}^{3}, 0\right),(u, v) \mapsto\left(3 u^{4}+u^{2} v, 4 u^{3}+2 u v, v\right)$ is the swallowtail (Figure $2)$, and $C:(\mathbb{R}, 0) \rightarrow\left(\mathbb{R}^{3}, 0\right), t \mapsto\left(t^{2} / 2, t^{3} / 3, t^{4} / 4\right)$ is the $(2,3,4)$-type (Figure 3 ).

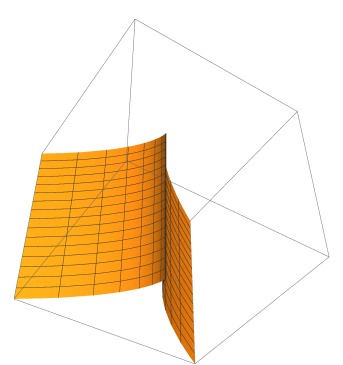

Figure 1. The cuspidal edge

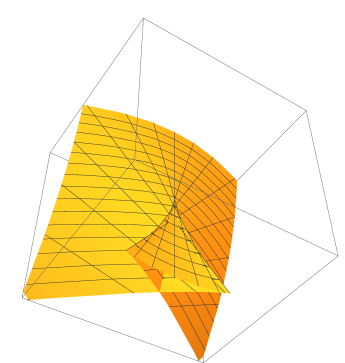

Figure 2. The swallowtail

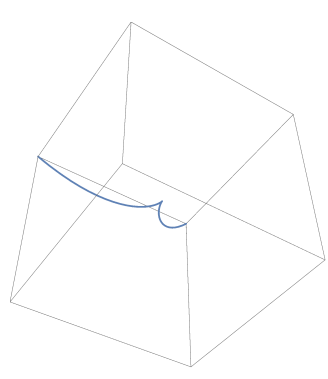

Figure 3 . The $(2,3,4)$-type

Proof. Since Remarks 3.3 and 3.8, it is enough to consider the Frenet type frame. By a direct calculation, we have

$$
\frac{\partial \mathcal{F} \mathcal{S}(\gamma)}{\partial t}(t, \lambda) \times \frac{\partial \mathcal{F} \mathcal{S}(\gamma)}{\partial \lambda}(t, \lambda)=-\left(\frac{\alpha(t) \dot{M}(t)-\dot{\alpha}(t) M(t)}{M^{2}(t)}+\lambda L(t)\right) \boldsymbol{\mu}(t)
$$

Therefore, $\left(t_{0}, \lambda_{0}\right) \in I \times \mathbb{R}$ is a singular point of $\mathcal{F S}(\gamma)$ if and only if

$$
\lambda_{0}=-\frac{\alpha\left(t_{0}\right) \dot{M}\left(t_{0}\right)-\dot{\alpha}\left(t_{0}\right) M\left(t_{0}\right)}{L\left(t_{0}\right) M^{2}\left(t_{0}\right)} .
$$

This means that (1) holds. By the above calculation, $\mathcal{F S}(\gamma): I \times \mathbb{R} \rightarrow \mathbb{R}^{3}$ is a wave front (cf. [1, 13]). The assertions (2) and (3) can be proven by using the criterion for the cuspidal edge $C E$ and the swallowtail $S W$ in [13]. We consider the signed density function $\Lambda: I \times \mathbb{R} \rightarrow \mathbb{R}$,

$$
\Lambda(t, \lambda)=\frac{\alpha(t) \dot{M}(t)-\dot{\alpha}(t) M(t)}{M^{2}(t)}+\lambda L(t) .
$$


Since $\Lambda_{\lambda}(t, \lambda)=L(t) \neq 0, \mathcal{F S}(\gamma)$ have only non-degenerate singular points. Moreover, the singular curve $c: I \rightarrow I \times \mathbb{R} \subset \mathbb{R}^{2}$ of $\mathcal{F} \mathcal{S}(\gamma)$ is given by

$$
c(t)=\left(t,-\frac{\alpha(t) \dot{M}(t)-\dot{\alpha}(t) M(t)}{L(t) M^{2}(t)}\right)
$$

and a null vector field $\eta: I \rightarrow \mathbb{R}^{2}$ along $c(t)$ is given by $\eta(t)=(1,-\alpha(t) L(t) / M(t))$.

On the other hand, by using the Frenet-Serret type formula of the evolute, we have

$$
\begin{aligned}
\dot{\mathcal{E}} v(\gamma)(t)= & \left(\frac{d}{d t}\left(\frac{\alpha(t) \dot{M}(t)-\dot{\alpha}(t) M(t)}{L(t) M^{2}(t)}\right)-\frac{\alpha(t) L(t)}{M(t)}\right) \boldsymbol{n}_{2}(t)=\alpha_{\mathcal{E} v}(t) \boldsymbol{n}_{2}(t), \\
\ddot{\mathcal{E}} v(\gamma)(t)= & -\alpha_{\mathcal{E} v}(t) L(t) \boldsymbol{n}_{1}(t)+\dot{\alpha}_{\mathcal{E} v}(t) \boldsymbol{n}_{2}(t), \\
\ddot{\mathcal{E}} v(\gamma)(t)= & -\alpha_{\mathcal{E} v}(t) L(t) M(t) \boldsymbol{\mu}(t)+\left(-2 \dot{\alpha}_{\mathcal{E} v}(t) L(t)-\alpha_{\mathcal{E} v}(t) \dot{L}(t)\right) \boldsymbol{n}_{1}(t) \\
& +\left(\ddot{\alpha}_{\mathcal{E} v}(t)-\alpha_{\mathcal{E} v}(t) L^{2}(t)\right) \boldsymbol{n}_{2}(t) .
\end{aligned}
$$

By using the criterion in [13], the focal surface $\mathcal{F} \mathcal{S}(\gamma)$ is locally diffeomorphic to the cuspidal edge $C E$ at $\left(t_{0}, \lambda_{0}\right)$ if and only if the condition (3.2) and

$$
\operatorname{det}\left(\dot{c}\left(t_{0}\right), \eta\left(t_{0}\right)\right)=\left.\frac{d}{d t}\left(\frac{\alpha(t) \dot{M}(t)-\dot{\alpha}(t) M(t)}{L(t) M^{2}(t)}\right)\right|_{t=t_{0}}-\frac{\alpha\left(t_{0}\right) L\left(t_{0}\right)}{M\left(t_{0}\right)}=\alpha_{\mathcal{E} v}\left(t_{0}\right) \neq 0
$$

holds. This condition is equivalent to $t_{0}$ is a regular point of $\mathcal{E} v(\gamma)$. Hence, we have the assertion (2).

Moreover, the focal surface $\mathcal{F S}(\gamma)$ is locally diffeomorphic to the swallowtail $S W$ at $\left(t_{0}, \lambda_{0}\right)$ if and only if the condition $(3.2), \operatorname{det}\left(\dot{c}\left(t_{0}\right), \eta\left(t_{0}\right)\right)=\alpha_{\mathcal{E} v}\left(t_{0}\right)=0$ and $(d / d t) \operatorname{det}(\dot{c}, \eta)\left(t_{0}\right)=\dot{\alpha}_{\mathcal{E} v}\left(t_{0}\right) \neq 0$ holds. On the other hand, the evolute $\mathcal{E} v(\gamma)$ is locally diffeomorphic to the $(2,3,4)$-type $C$ at $t_{0}$ if and only if $\dot{\mathcal{E}} v(\gamma)\left(t_{0}\right)=0$ and $\operatorname{rank}\left(\ddot{\mathcal{E}} v(\gamma)\left(t_{0}\right), \ddot{\mathcal{E}} v(\gamma)\left(t_{0}\right)\right)=2$, that is, $\alpha_{\mathcal{E} v}\left(t_{0}\right)=0$ and $\dot{\alpha}_{\mathcal{E} v}\left(t_{0}\right) \neq 0$. It follows that (3) holds. This completes the proof of Theorem.

\section{Properties of the evolutes of framed immersions}

In this section we investigate some properties of the evolute of a framed immersion.

\subsection{Support functions}

Let $\left(\gamma, \boldsymbol{n}_{1}, \boldsymbol{n}_{2}\right): I \rightarrow \mathbb{R}^{3} \times \Delta$ be a framed immersion with $f(t)=L(t) M^{2}(t) \neq 0$, where $\left\{\boldsymbol{n}_{1}(t), \boldsymbol{n}_{2}(t), \boldsymbol{\mu}(t)\right\}$ is the Frenet type frame. We define a family of functions $F_{\mu}: I \times \mathbb{R}^{3} \rightarrow \mathbb{R}, F_{\mu}(t, \boldsymbol{x})=(\gamma(t)-\boldsymbol{x}) \cdot \boldsymbol{\mu}(t)$. We call $F_{\mu}$ a support function of $\gamma$ with respect to $\boldsymbol{\mu}$ (cf. [12]). We denote $F_{\mu}\left(\boldsymbol{x}_{0}\right)(t)=F_{\mu}\left(t, \boldsymbol{x}_{0}\right)$ for any $\boldsymbol{x}_{0} \in \mathbb{R}^{3}$.

By a direct calculation, we have the following proposition.

Proposition 4.1. Under the above notations, we have the following:

(1) $F_{\mu}\left(\boldsymbol{x}_{0}\right)\left(t_{0}\right)=0$ if and only if there exist $\lambda_{1}, \lambda_{2} \in \mathbb{R}$ such that $\gamma\left(t_{0}\right)-\boldsymbol{x}_{0}=$ $\lambda_{1} \boldsymbol{n}_{1}\left(t_{0}\right)+\lambda_{2} \boldsymbol{n}_{2}\left(t_{0}\right)$. 
(2) $F_{\mu}\left(\boldsymbol{x}_{0}\right)\left(t_{0}\right)=\dot{F}_{\mu}\left(\boldsymbol{x}_{0}\right)\left(t_{0}\right)=0$ if and only if there exists $\lambda \in \mathbb{R}$ such that

$$
\gamma\left(t_{0}\right)-\boldsymbol{x}_{0}=\frac{\alpha\left(t_{0}\right)}{M\left(t_{0}\right)} \boldsymbol{n}_{1}\left(t_{0}\right)+\lambda \boldsymbol{n}_{2}\left(t_{0}\right) .
$$

(3) $F_{\mu}\left(\boldsymbol{x}_{0}\right)\left(t_{0}\right)=\dot{F}_{\mu}\left(\boldsymbol{x}_{0}\right)\left(t_{0}\right)=\ddot{F}_{\mu}\left(\boldsymbol{x}_{0}\right)\left(t_{0}\right)=0$ if and only if

$$
\gamma\left(t_{0}\right)-\boldsymbol{x}_{0}=\frac{\alpha\left(t_{0}\right)}{M\left(t_{0}\right)} \boldsymbol{n}_{1}\left(t_{0}\right)-\frac{\alpha\left(t_{0}\right) \dot{M}\left(t_{0}\right)-\dot{\alpha}\left(t_{0}\right) M\left(t_{0}\right)}{L\left(t_{0}\right) M^{2}\left(t_{0}\right)} \boldsymbol{n}_{2}\left(t_{0}\right)
$$

For the support function of $\gamma$ with respect to $\boldsymbol{\mu}$, the discriminant set

$$
\mathcal{D}_{F_{\mu}}=\left\{\boldsymbol{x} \in \mathbb{R}^{3} \mid \text { there exists } t \in I \text { such that } F_{\mu}=\frac{\partial F_{\mu}}{\partial t}=0 \text { at }(t, \boldsymbol{x})\right\}
$$

coincides with the image of the focal surface $\mathcal{F S}(\gamma)$. Moreover, the secondary discriminant set

$$
\mathcal{D}_{F_{\mu}}^{2}=\left\{\boldsymbol{x} \in \mathbb{R}^{3} \mid \text { there exists } t \in I \text { such that } F_{\mu}=\frac{\partial F_{\mu}}{\partial t}=\frac{\partial^{2} F_{\mu}}{\partial t^{2}}=0 \text { at }(t, \boldsymbol{x})\right\}
$$

coincides with the image of the evolute $\mathcal{E} v(\gamma)$.

\subsection{Parallel curves}

We consider parallel curves of a framed curve and its evolute. Let $\theta: I \rightarrow \mathbb{R}$ be a smooth function with $\dot{\theta}(t)=\ell(t)$ for all $t \in I$. The parallel curve $\gamma_{(a, b)}: I \rightarrow \mathbb{R}^{3}$ of a framed curve $\left(\gamma, \nu_{1}, \nu_{2}\right)$ is defined by

$$
\gamma_{(a, b)}(t)=\gamma(t)+(a \cos \theta(t)+b \sin \theta(t)) \nu_{1}(t)+(-a \sin \theta(t)+b \cos \theta(t)) \nu_{2}(t),
$$

where $a, b \in \mathbb{R}$. Then we have the following results.

Proposition 4.2. The parallel curve $\gamma_{(a, b)}$ is a framed base curve. More precisely, $\left(\gamma_{(a, b)}, \nu_{1}, \nu_{2}\right): I \rightarrow \mathbb{R}^{3} \times \Delta$ is a framed curve with the curvature $(\ell(t), m(t), n(t), \alpha(t)+$ $a(m(t) \cos \theta(t)-n(t) \sin \theta(t))+b(m(t) \sin \theta(t)+n(t) \cos \theta(t)))$.

Proposition 4.3. $\left(\gamma_{(a, b)}, \nu_{1}, \nu_{2}\right): I \rightarrow \mathbb{R}^{3} \times \Delta$ is a framed immersion with $f_{(a, b)}(t) \neq$ 0 and $\mathcal{E} v\left(\gamma_{(a, b)}\right)(t)=\mathcal{E} v(\gamma)(t)$ for any $a, b \in \mathbb{R}$.

Proof. By Proposition 4.2, $\left(\gamma_{(a, b)}, \nu_{1}, \nu_{2}\right): I \rightarrow \mathbb{R}^{3} \times \Delta$ is also a framed curve and we have $f_{(a, b)}(t)=f(t) \neq 0$ for all $t \in I$. It follows that $\left(\gamma_{(a, b)}, \nu_{1}, \nu_{2}\right)$ is a framed immersion. By a direct calculation, we have $\mathcal{E} v\left(\gamma_{(a, b)}\right)(t)=\mathcal{E} v(\gamma)(t)$ for any $a, b \in \mathbb{R}$.

Proposition 4.4. The set of singular values of parallel curves $\gamma_{(a, b)}$ is contained in the image of the focal surface $\mathcal{F} \mathcal{S}(\gamma)$ for any $a, b \in \mathbb{R}$.

Proof. We put $A_{(a, b)}(t)=a \cos \theta(t)+b \sin \theta(t)$ and $B_{(a, b)}(t)=-a \sin \theta(t)+b \cos \theta(t)$, where $\dot{\theta}(t)=\ell(t)$ for all $t \in I$. Then we have $\gamma_{(a, b)}(t)=\gamma(t)+A_{(a, b)}(t) \nu_{1}(t)+$ $B_{(a, b)}(t) \nu_{2}(t)$. If $t_{0}$ is a singular point of $\gamma_{(a, b)}$, then we have $\alpha\left(t_{0}\right)+m\left(t_{0}\right) A_{(a, b)}\left(t_{0}\right)+$ $n\left(t_{0}\right) B_{(a, b)}\left(t_{0}\right)=0$. This means that $\gamma_{(a, b)}\left(t_{0}\right) \in \mathcal{F S}(\gamma)(I \times J)$ for any $a, b \in \mathbb{R}$. 


\subsection{Evolutes of framed immersions and spheres}

For a Frenet curve $\gamma: I \rightarrow \mathbb{R}^{3}$ with $\tau(t) \neq 0,(d / d t) E v(\gamma)(t)=0$ for all $t \in I$ if and only if there exist a constant vector $c \in \mathbb{R}^{3}$ and a positive real number $r \in \mathbb{R}$ such that $\gamma(t) \in S^{2}(\boldsymbol{c}, r)$ for all $t \in I$, where $S^{2}(\boldsymbol{c}, r)=\left\{\boldsymbol{x} \in \mathbb{R}^{3}|| \boldsymbol{x}-\boldsymbol{c} \mid=r\right\}$ (cf. $[3,8])$. We consider the relationships between the evolute of a framed immersion and a sphere. Let $\left(\gamma, \nu_{1}, \nu_{2}\right): I \rightarrow \mathbb{R}^{3} \times \Delta$ be a framed immersion with $f(t) \neq 0$.

Proposition 4.5. If $(d / d t) \mathcal{E} v(\gamma)(t)=0$ for all $t \in I$, then there exist a constant vector $\boldsymbol{c} \in \mathbb{R}^{3}$ and a non-negative real number $r \in \mathbb{R}$ such that $\gamma(t) \in S^{2}(\boldsymbol{c}, r)$ for all $t \in I$.

Proof. Since $(d / d t) \mathcal{E} v(\gamma)(t)=0$ for all $t \in I$, we put $\boldsymbol{c}=\mathcal{E} v(\gamma)(t)$. By the equation (3.1) in Proposition 3.4, we have $(d / d t)\left(|\gamma(t)-\boldsymbol{c}|^{2}\right)=0$ for all $t \in I$. Hence there exists a non-negative real number $r=|\gamma(t)-\boldsymbol{c}|$ such that $\gamma(t) \in S^{2}(\boldsymbol{c}, r)$ for all $t \in I$.

Lemma 4.6. $(d / d t) \mathcal{E} v(\gamma)(t)=0$ for all $t \in I$ if and only if there exist functions $u, v: I \rightarrow \mathbb{R}$ and a constant $\boldsymbol{c} \in \mathbb{R}^{3}$ such that $\gamma(t)-\boldsymbol{c}=u(t) \nu_{1}(t)+v(t) \nu_{2}(t)$ for all $t \in I$.

Proof. If $(d / d t) \mathcal{E} v(\gamma)(t)=0$ for all $t \in I$, then we can write $\gamma(t)-\boldsymbol{c}=u(t) \nu_{1}(t)+$ $v(t) \nu_{2}(t)$, where $\boldsymbol{c}=\mathcal{E} v(\gamma)(t), u(t)=(\alpha(t) \dot{n}(t)-\dot{\alpha}(t) n(t)+\alpha(t) \ell(t) m(t)) / f(t)$ and $v(t)=-(\alpha(t) \dot{m}(t)-\dot{\alpha}(t) m(t)-\alpha(t) \ell(t) n(t)) / f(t)$.

Conversely, there exist functions $u, v: I \rightarrow \mathbb{R}$ and a constant $c \in \mathbb{R}^{3}$ such that $\gamma(t)-\boldsymbol{c}=u(t) \nu_{1}(t)+v(t) \nu_{2}(t)$ for all $t \in I$. By differentiating, we have

$\alpha(t) \boldsymbol{\mu}(t)=(\dot{u}(t)-v(t) \ell(t)) \nu_{1}(t)+(\dot{v}(t)+u(t) \ell(t)) \nu_{2}(t)+(u(t) m(t)+v(t) n(t)) \boldsymbol{\mu}(t)$.

Since $\left\{\nu_{1}(t), \nu_{2}(t), \boldsymbol{\mu}(t)\right\}$ is an orthonormal frame, we have $\dot{u}(t)-v(t) \ell(t)=0$, $\dot{v}(t)+u(t) \ell(t)=0, \alpha(t)=u(t) m(t)+v(t) n(t)$ for all $t \in I$. Thus $\dot{\alpha}(t)=\dot{u}(t) m(t)+$ $u(t) \dot{m}(t)+\dot{v}(t) n(t)+v(t) \dot{n}(t)$. By a direct calculation, we have $\alpha(t) \dot{n}(t)-\dot{\alpha}(t) n(t)+$ $\alpha(t) \ell(t) m(t)=u(t) f(t), \alpha(t) \dot{m}(t)-\dot{\alpha}(t) m(t)-\alpha(t) \ell(t) n(t)=-v(t) f(t)$. Hence

$$
\alpha_{\mathcal{E} v}(t)=\frac{1}{\sqrt{m^{2}(t)+n^{2}(t)}}(n(t)(\dot{u}(t)-v(t) \ell(t))-m(t)(\dot{v}(t)+u(t) \ell(t)))=0
$$

for all $t \in I$. It follows that $(d / d t) \mathcal{E} v(\gamma)(t)=0$ for all $t \in I$.

Proposition 4.7. Suppose that the set of regular points of $\gamma$ is dense in I. Then $(d / d t) \mathcal{E} v(\gamma)(t)=0$ for all $t \in I$ if and only if there exist a constant vector $\boldsymbol{c} \in \mathbb{R}^{3}$ and a positive real number $r \in \mathbb{R}$ such that $\gamma(t) \in S^{2}(\boldsymbol{c}, r)$ for all $t \in I$.

Proof. Suppose that $(d / d t) \mathcal{E} v(\gamma)(t)=0$ for all $t \in I$. By Proposition 4.5 , there exist a constant vector $c \in \mathbb{R}^{3}$ and a non-negative real number $r \in \mathbb{R}$ such that $\gamma(t) \in S^{2}(\boldsymbol{c}, r)$. If $r=0$, then $\gamma(t)=\boldsymbol{c}$ and hence $\dot{\gamma}(t)=0$ for all $t \in I$. Since the set of regular points of $\gamma$ is dense in $I$, the case does not occur. It follows that $r$ is positive.

Conversely, suppose that there exist a constant vector $c \in \mathbb{R}^{3}$ and a positive real number $r \in \mathbb{R}$ such that $\gamma(t) \in S^{2}(\boldsymbol{c}, r)$. By the assumption, we have $|\gamma(t)-\boldsymbol{c}|^{2}=r^{2}$. 
It follows that $\alpha(t) \boldsymbol{\mu}(t) \cdot(\gamma(t)-\boldsymbol{c})=0$. Since the set of regular points of $\gamma$ is dense in $I$, we have $\boldsymbol{\mu}(t) \cdot(\gamma(t)-\boldsymbol{c})=0$ for all $t \in I$. Then there exist functions $u, v: I \rightarrow \mathbb{R}$ and a constant $\boldsymbol{c} \in \mathbb{R}^{3}$ such that $\gamma(t)-\boldsymbol{c}=u(t) \nu_{1}(t)+v(t) \nu_{2}(t)$ for all $t \in I$. By Lemma 4.6, we have $(d / d t) \mathcal{E} v(\gamma)(t)=0$ for all $t \in I$.

\subsection{Contact between framed immersions and evolutes}

We consider contact between framed immersions and its evolutes. We recall the notion of the contact between framed curves, see [10]. Let $\left(\gamma, \nu_{1}, \nu_{2}\right): I \rightarrow \mathbb{R}^{3} \times$ $\Delta, t \mapsto\left(\gamma(t), \nu_{1}(t), \nu_{2}(t)\right)$ and $\left(\widetilde{\gamma}, \widetilde{\nu}_{1}, \widetilde{\nu}_{2}\right): \widetilde{I} \rightarrow \mathbb{R}^{3} \times \Delta, u \mapsto\left(\widetilde{\gamma}(u), \widetilde{\nu}_{1}(u), \widetilde{\nu}_{2}(u)\right)$ be framed curves with the curvatures $(\ell(t), m(t), n(t), \alpha(t))$ and $(\widetilde{\ell}(u), \widetilde{m}(u), \widetilde{n}(u), \widetilde{\alpha}(u))$, respectively. Let $k$ be a natural number. We denote $\mathcal{F}(t)=(\ell(t), m(t), n(t), \alpha(t))$ and $\widetilde{\mathcal{F}}(u)=(\widetilde{\ell}(u), \widetilde{m}(u), \widetilde{n}(u), \widetilde{\alpha}(u))$ for convenience. We say that $\left(\gamma, \nu_{1}, \nu_{2}\right)$ and $\left(\widetilde{\gamma}, \widetilde{\nu}_{1}, \widetilde{\nu}_{2}\right)$ have at least $k$-th order contact at $t=t_{0}, u=u_{0}$ if $d^{i}\left(\gamma, \nu_{1}, \nu_{2}\right) / d t^{i}\left(t_{0}\right)=$ $d^{i}\left(\widetilde{\gamma}, \widetilde{\nu}_{1}, \widetilde{\nu}_{2}\right) / d u^{i}\left(u_{0}\right)$, for $i=0,1, \ldots, k-1$. In general, we may assume that $\left(\gamma, \nu_{1}, \nu_{2}\right)$ and $\left(\widetilde{\gamma}, \widetilde{\nu}_{1}, \widetilde{\nu}_{2}\right)$ have at least first order contact at any point $t=t_{0}, u=u_{0}$, up to congruence as framed curves.

Theorem 4.8. ([10]) If $\left(\gamma, \nu_{1}, \nu_{2}\right)$ and $\left(\widetilde{\gamma}, \widetilde{\nu}_{1}, \widetilde{\nu}_{2}\right)$ have at least $(k+1)$-th order contact at $t=t_{0}, u=u_{0}$ then

$$
\left(d^{i} \mathcal{F} / d t^{i}\right)\left(t_{0}\right)=\left(d^{i} \widetilde{\mathcal{F}} / d u^{i}\right)\left(u_{0}\right),
$$

for $i=0,1, \ldots, k-1$. Conversely, if conditions (4.1) hold, then $\left(\gamma, \nu_{1}, \nu_{2}\right)$ and $\left(\widetilde{\gamma}, \widetilde{\nu}_{1}, \widetilde{\nu}_{2}\right)$ have at least $(k+1)$-th order contact at $t=t_{0}, u=u_{0}$, up to congruence as framed curves.

By using the induction, we have the following Proposition, in detail see [11].

Proposition 4.9. Let $\left(\gamma, \nu_{1}, \nu_{2}\right): I \rightarrow \mathbb{R}^{3} \times \Delta$, $t \mapsto\left(\gamma(t), \nu_{1}(t), \nu_{2}(t)\right)$ and $\left(\widetilde{\gamma}, \widetilde{\nu}_{1}, \widetilde{\nu}_{2}\right): \widetilde{I} \rightarrow \mathbb{R}^{3} \times \Delta, u \mapsto\left(\widetilde{\gamma}(u), \widetilde{\nu}_{1}(u), \widetilde{\nu}_{2}(u)\right)$ be framed immersions with $f(t) \neq 0$ and $\widetilde{f}(u) \neq 0$, respectively. If $\left(\gamma, \nu_{1}, \nu_{2}\right)$ and $\left(\widetilde{\gamma}, \widetilde{\nu}_{1}, \widetilde{\nu}_{2}\right)$ have at least $(k+2)$ th order contact at $t=t_{0}, u=u_{0}$ for $k \in \mathbb{N}$, then $\left(\mathcal{E} v(\gamma), \boldsymbol{\mu}, \boldsymbol{n}_{1}\right)$ and $\left(\mathcal{E} v(\widetilde{\gamma}), \widetilde{\boldsymbol{\mu}}, \widetilde{\boldsymbol{n}}_{1}\right)$ have at least $k$-th order contact at $t=t_{0}, u=u_{0}$.

Proposition 4.10. Let $\left(\gamma, \boldsymbol{n}_{1}, \boldsymbol{n}_{2}\right): I \rightarrow \mathbb{R}^{3} \times \Delta$ be a framed immersion with $f(t)=L(t) M^{2}(t) \neq 0$, where $\left\{\boldsymbol{n}_{1}(t), \boldsymbol{n}_{2}(t), \boldsymbol{\mu}(t)\right\}$ is the Frenet type frame. Then we have the following:

(1) There exists a rotated frame $\left\{\overline{\boldsymbol{\mu}}(t), \overline{\boldsymbol{n}}_{1}(t), \boldsymbol{n}_{2}(t)\right\}$ along $\mathcal{E} v(\gamma)(t)$ by $\theta(t)$ such that $\left(\gamma, \boldsymbol{n}_{1}, \boldsymbol{n}_{2}\right)$ and $\left(\mathcal{E} v(\gamma), \overline{\boldsymbol{\mu}}, \overline{\boldsymbol{n}}_{1}\right)$ are congruent as framed immersions if and only if $L(t)=-M(t)$ and $\alpha(t)=\alpha_{\mathcal{E} v}(t)$ for all $t \in I$.

(2) There exists a reflected frame $\left\{\widetilde{\boldsymbol{\mu}}(t), \widetilde{\boldsymbol{n}}_{1}(t),-\boldsymbol{n}_{2}(t)\right\}$ along $\mathcal{E} v(\gamma)(t)$ by $\theta(t)$ such that $\left(\gamma, \boldsymbol{n}_{1}, \boldsymbol{n}_{2}\right)$ and $\left(\mathcal{E} v(\gamma), \widetilde{\boldsymbol{\mu}}, \widetilde{\boldsymbol{n}}_{1}\right)$ are congruent as framed immersions if and only if $L(t)=M(t)$ and $\alpha(t)=-\alpha_{\mathcal{E} v}(t)$ for all $t \in I$.

Proof. Suppose that there exists a rotated frame $\left\{\overline{\boldsymbol{\mu}}(t), \overline{\boldsymbol{n}}_{1}(t), \boldsymbol{n}_{2}(t)\right\}$ along $\mathcal{E} v(\gamma)(t)$ by $\theta(t)$ such that $\left(\gamma, \boldsymbol{n}_{1}, \boldsymbol{n}_{2}\right)$ and $\left(\mathcal{E} v(\gamma), \overline{\boldsymbol{\mu}}, \overline{\boldsymbol{n}}_{1}\right)$ are congruent as framed curves. By Theorem 2.5 and Proposition 3.4, we have

$$
(L(t), M(t), 0, \alpha(t))=\left(-M(t)-\dot{\theta}(t),-L(t) \sin \theta(t), L(t) \cos \theta(t), \alpha_{\mathcal{E} v}(t)\right)
$$


Evolutes and focal surfaces of framed immersions in the Euclidean space 13 for all $t \in I$. Since $L(t) \cos \theta(t)=0$ and $L(t) \neq 0$ for all $t \in I$, we have $\cos \theta(t)=0$ and $\dot{\theta}(t)=0$. If $\sin \theta(t)=-1$, then $L(t)=-M(t)$ and $M(t)=L(t)$. It follows that $L(t)=M(t)=0$. Since $f(t) \neq 0$, the case does not occur. It follows that $\sin \theta(t)=1$. Hence, we have $L(t)=-M(t)$ and $\alpha(t)=\alpha_{\mathcal{E} v}(t)$ for all $t \in I$.

Conversely, suppose that $L(t)=-M(t)$ and $\alpha(t)=\alpha_{\mathcal{E} v}(t)$ for all $t \in I$. We take a rotated frame $\left\{\overline{\boldsymbol{\mu}}(t), \overline{\boldsymbol{n}}_{1}(t), \boldsymbol{n}_{2}(t)\right\}$ along $\mathcal{E} v(\gamma)(t)$ by $\theta(t)=\pi / 2$. Then, the curvature of $\left(\mathcal{E} v(\gamma), \overline{\boldsymbol{\mu}}(t), \overline{\boldsymbol{n}}_{1}(t)\right)$ is given by $\left(-M(t),-L(t), 0, \alpha_{\mathcal{E} v}(t)\right)$. By Theorem $2.5,\left(\gamma, \boldsymbol{n}_{1}, \boldsymbol{n}_{2}\right)$ and $\left(\mathcal{E} v(\gamma), \overline{\boldsymbol{\mu}}, \overline{\boldsymbol{n}}_{1}\right)$ are congruent as framed curves. Therefore the assertion (1) holds.

By the similar arguments to the above, we have the assertion (2).

Remark 4.11. A function $L(t) / M(t)$ is constant if and only if $\gamma$ is a framed helix, see [9]. Therefore, if $M(t)= \pm L(t)$ for all $t \in I$, then $\gamma$ is a framed helix.

\subsection{The $k$-th evolutes of framed immersions}

In this subsection we consider repeated evolutes of framed immersions. By Proposition 3.4, we have $f_{\mathcal{E} v}(t)=-L^{2}(t) M(t) \neq 0$. It follows that the evolute $\mathcal{E} v(\gamma)$ has the evolute $\mathcal{E} v(\mathcal{E} v(\gamma))(t)$. We denote $\mathcal{E} v^{0}(\gamma)(t)=\gamma(t), \mathcal{E} v^{1}(\gamma)(t)=\mathcal{E} v(\gamma)(t)$, $\left\{\nu_{1}^{1}(t), \nu_{2}^{1}(t), \boldsymbol{\mu}^{1}(t)\right\}=\left\{\boldsymbol{\mu}(t), \boldsymbol{n}_{1}(t), \boldsymbol{n}_{2}(t)\right\}$ and $\left(\ell_{1}(t), m_{1}(t), n_{1}(t), \alpha_{1}(t)\right)=(-M(t)$, $\left.0, L(t), \alpha_{\mathcal{E} v}(t)\right)$ and for convenience. We give the form of the $k$-th evolute of the framed immersion, where $k$ is a natural number greater than or equal to 2 . We define $\mathcal{E} v^{k}(\gamma)(t)=\mathcal{E} v\left(\mathcal{E} v^{k-1}(\gamma)\right)(t)$ and

$$
\begin{gathered}
\nu_{1}^{k}(t)=\left\{\begin{array}{ll}
\nu_{1}^{1}(t) & (k: \text { odd }) \\
\boldsymbol{\mu}^{1}(t) & (k: \text { even })
\end{array}, \nu_{2}^{k}(t)=\left\{\begin{array}{cc}
\nu_{2}^{1}(t) & (k: \text { odd }) \\
-\nu_{2}^{1}(t) & (k: \text { even })
\end{array}, \boldsymbol{\mu}^{k}(t)=\nu_{1}^{k}(t) \times \nu_{2}^{k}(t),\right.\right. \\
\ell_{k}(t)=\left\{\begin{array}{ll}
\ell_{1}(t) & (k: \text { odd }) \\
n_{1}(t) & (k: \text { even })
\end{array}, m_{k}(t)=0, n_{k}(t)=\left\{\begin{array}{cc}
n_{1}(t) & (k: \text { odd }) \\
\ell_{1}(t) & (k: \text { even })
\end{array},\right.\right. \\
\alpha_{k}(t)=-\frac{d}{d t}\left(\frac{\alpha_{k-1}(t) \dot{n}_{k-1}(t)-\dot{\alpha}_{k-1}(t) n_{k-1}(t)}{\ell_{k-1}(t) n_{k-1}^{2}(t)}\right)+\frac{\alpha_{k-1}(t) \ell_{k-1}(t)}{n_{k-1}(t)}
\end{gathered}
$$

inductively.

Theorem 4.12. Let $\left(\gamma, \nu_{1}, \nu_{2}\right): I \rightarrow \mathbb{R}^{3} \times \Delta$ be a framed immersion with $f(t) \neq 0$ and $k \geq 2$. Then $\left(\mathcal{E} v^{k}(\gamma), \nu_{1}^{k}, \nu_{2}^{k}\right): I \rightarrow \mathbb{R}^{3} \times \Delta$ is a framed immersion with the curvature $\left(\ell_{k}, m_{k}, n_{k}, \alpha_{k}\right)$, where the $k$-th evolute of the framed immersion is given by

$\mathcal{E} v^{k}(\gamma)(t)=\mathcal{E} v^{k-1}(\gamma)(t)-\frac{\alpha_{k-1}(t) \dot{n}_{k-1}(t)-\dot{\alpha}_{k-1}(t) n_{k-1}(t)}{\ell_{k-1}(t) n_{k-1}^{2}(t)} \nu_{1}^{k-1}(t)-\frac{\alpha_{k-1}(t)}{n_{k-1}(t)} \nu_{2}^{k-1}(t)$.

Proof. We give a proof by using the induction on $k$. First we consider the case of $k=2$. By the definition of the evolute,

$$
\mathcal{E} v^{2}(\gamma)(t)=\mathcal{E} v^{1}(\gamma)(t)-\frac{\alpha_{1}(t) \dot{n}_{1}(t)-\dot{\alpha}_{1}(t) n_{1}(t)}{\ell_{1}(t) n_{1}^{2}(t)} \nu_{1}^{1}(t)-\frac{\alpha_{1}(t)}{n_{1}(t)} \nu_{2}^{1}(t)
$$


Since

$$
\frac{d}{d t} \mathcal{E} v^{2}(\gamma)(t)=\left(-\frac{d}{d t}\left(\frac{\alpha_{1}(t) \dot{n}_{1}(t)-\dot{\alpha}_{1}(t) n_{1}(t)}{\ell_{1}(t) n_{1}^{2}(t)}\right)+\frac{\alpha_{1}(t) \ell_{1}(t)}{n_{1}(t)}\right) \nu_{1}^{1}(t),
$$

we have $\mathcal{E} \dot{v}^{2}(\gamma)(t) \cdot \boldsymbol{\mu}^{1}(t)=0$ and $\dot{\mathcal{E}} \dot{v}^{2}(\gamma)(t) \cdot\left(-\nu_{2}^{1}(t)\right)=0$. Hence, $\left(\mathcal{E} v^{2}(\gamma), \nu_{1}^{2}, \nu_{2}^{2}\right)$ is a framed immersion. Moreover, we have $\ell_{2}(t)=n_{1}(t), m_{2}(t)=0, n_{2}(t)=\ell_{1}(t)$ and

$$
\alpha_{2}(t)=-\frac{d}{d t}\left(\frac{\alpha_{1}(t) \dot{n}_{1}(t)-\dot{\alpha}_{1}(t) n_{1}(t)}{\ell_{1}(t) n_{1}^{2}(t)}\right)+\frac{\alpha_{1}(t) \ell_{1}(t)}{n_{1}(t)} .
$$

The assertion in the case of $k=2$ holds.

Suppose that the assumption holds for the case of the $k$-th evolute. By the definition,

$$
\mathcal{E} v^{k+1}(\gamma)(t)=\mathcal{E} v^{k}(\gamma)(t)-\frac{\alpha_{k}(t) \dot{n}_{k}(t)-\dot{\alpha}_{k}(t) n_{k}(t)}{\ell_{k}(t) n_{k}^{2}(t)} \nu_{1}^{k}(t)-\frac{\alpha_{k}(t)}{n_{k}(t)} \nu_{2}^{k}(t) .
$$

Since

$$
\frac{d}{d t} \mathcal{E} v^{k+1}(\gamma)(t)=\left(-\frac{d}{d t}\left(\frac{\alpha_{k}(t) \dot{n}_{k}(t)-\dot{\alpha}_{k}(t) n_{k}(t)}{\ell_{k}(t) n_{k}^{2}(t)}\right)+\frac{\alpha_{k}(t) \ell_{k}(t)}{n_{k}(t)}\right) \nu_{1}^{k}(t),
$$

we have $\dot{\mathcal{E} v^{k+1}}(\gamma)(t) \cdot \boldsymbol{\mu}^{k}(t)=0$ and $\dot{\mathcal{E}}^{k+1}(\gamma)(t) \cdot\left(-\nu_{2}^{k}(t)\right)=0$. It follows that $\left(\mathcal{E} v^{k+1}(\gamma), \nu_{1}^{k+1}, \nu_{2}^{k+1}\right)$ is a framed immersion. Moreover, by a direct calculation, the curvature is given by $\ell_{k+1}(t)=n_{k}(t), m_{k+1}(t)=0, n_{k+1}(t)=\ell_{k}(t)$ and $\alpha_{k+1}(t)$.

By the similar argument to the proof of Proposition 4.10, we have the following proposition.

Proposition 4.13. Let $\left(\gamma, \boldsymbol{n}_{1}, \boldsymbol{n}_{2}\right): I \rightarrow \mathbb{R}^{3} \times \Delta$ be a framed immersion with $f(t)=L(t) M^{2}(t) \neq 0$, where $\left\{\boldsymbol{n}_{1}(t), \boldsymbol{n}_{2}(t), \boldsymbol{\mu}(t)\right\}$ is the Frenet type frame.

(1) Suppose that $k$ is an odd number.

(a) There exists a rotated frame $\left\{\overline{\boldsymbol{\mu}}(t), \overline{\boldsymbol{n}}_{1}(t), \boldsymbol{n}_{2}(t)\right\}$ along $\mathcal{E} v^{k}(\gamma)(t)$ by $\theta(t)$ such that $\left(\gamma, \boldsymbol{n}_{1}, \boldsymbol{n}_{2}\right)$ and $\left(\mathcal{E} v^{k}(\gamma), \overline{\boldsymbol{\mu}}, \overline{\boldsymbol{n}}_{1}\right)$ are congruent as framed immersions if and only if $L(t)=-M(t)$ and $\alpha(t)=\alpha_{k}(t)$ for all $t \in I$.

(b) There exists a reflected frame $\left\{\widetilde{\boldsymbol{\mu}}(t), \widetilde{\boldsymbol{n}}_{1}(t),-\boldsymbol{n}_{2}(t)\right\}$ along $\mathcal{E} v^{k}(\gamma)(t)$ by $\theta(t)$ such that $\left(\gamma, \boldsymbol{n}_{1}, \boldsymbol{n}_{2}\right)$ and $\left(\mathcal{E} v^{k}(\gamma), \widetilde{\boldsymbol{\mu}}, \widetilde{\boldsymbol{n}}_{1}\right)$ are congruent as framed immersions if and only if $L(t)=M(t)$ and $\alpha(t)=-\alpha_{k}(t)$ for all $t \in I$.

(2) Suppose that $k$ is an even number.

(c) There exists a rotated frame $\left\{\overline{\boldsymbol{n}}_{2}(t),-\overline{\boldsymbol{n}}_{1}(t), \boldsymbol{\mu}(t)\right\}$ along $\mathcal{E} v^{k}(\gamma)(t)$ by $\theta(t)$ such that $\left(\gamma, \boldsymbol{n}_{1}, \boldsymbol{n}_{2}\right)$ and $\left(\mathcal{E} v^{k}(\gamma), \overline{\boldsymbol{n}}_{2},-\overline{\boldsymbol{n}}_{1}\right)$ are congruent as framed immersions if and only if $\alpha(t)=\alpha_{k}(t)$ for all $t \in I$,

(d) There does not exist a reflected frame $\left\{\widetilde{\boldsymbol{n}}_{2}(t),-\widetilde{\boldsymbol{n}}_{1}(t),-\boldsymbol{\mu}(t)\right\}$ along $\mathcal{E} v^{k}(\gamma)(t)$ by $\theta(t)$ such that $\left(\gamma, \boldsymbol{n}_{1}, \boldsymbol{n}_{2}\right)$ and $\left(\mathcal{E} v^{k}(\gamma), \widetilde{\boldsymbol{n}}_{2},-\widetilde{\boldsymbol{n}}_{1}\right)$ are congruent as framed immersions. 


\section{Examples}

We give examples to understand the phenomena for evolutes and focal surfaces of framed immersions.

Example 5.1. Let $n$ be a natural number greater than or equal to 2 and let $\left(\gamma, \nu_{1}, \nu_{2}\right): \mathbb{R} \rightarrow \mathbb{R}^{3} \times \Delta$ be $\gamma(t)=\left(t^{n} / n, t^{n+1} /(n+1), t^{n+2} /(n+2)\right), \nu_{1}(t)=$ $(-t, 1,0) / \sqrt{1+t^{2}}, \nu_{2}(t)=\left(-t^{2},-t^{3}, 1+t^{2}\right) / \sqrt{\left(1+t^{2}\right)\left(1+t^{2}+t^{4}\right)}$. By a direct calculation, we have $f(t)=2 /\left(1+t^{2}+t^{4}\right)^{\frac{3}{2}} \neq 0$. The evolute and focal surface are given by

$$
\begin{aligned}
\mathcal{E} v(\gamma)(t)= & \left((n-2)(n-1)+n^{2} t^{2}+n(n+3) t^{4}\right) t^{n} /(2 n), \\
& -\left((n-2)(n+1)+n(n+2) t^{2}+(n+1)(n+4) t^{4}\right) t^{n-1} /(n+1), \\
& \left.\left((n-1)(n+2)+(n+2)^{2} t^{2}+(n+3)(n+4) t^{4}\right) t^{n-2} /(2(n+2))\right), \\
\mathcal{F} \mathcal{S}(\gamma)(t, \lambda)=( & t^{n} / n-\left(1+t^{2}+t^{4}\right) t^{n}-\lambda t^{2} \sqrt{\left(1+t^{2}\right) /\left(1+t^{2}+t^{4}\right)}, \\
& t^{n+1} /(n+1)+\left(1+t^{2}+t^{4}\right) t^{n-1}+2 \lambda t \sqrt{\left(1+t^{2}\right) /\left(1+t^{2}+t^{4}\right)} \\
& \left.t^{n+2} /(n+2)-\lambda \sqrt{\left(1+t^{2}\right) /\left(1+t^{2}+t^{4}\right)}\right) .
\end{aligned}
$$

In the case of $n=2$, the $(2,3,4)$-type (blue curve), the evolute (red curve) and the focal surface of the $(2,3,4)$-type see Figures 4 and 5 .

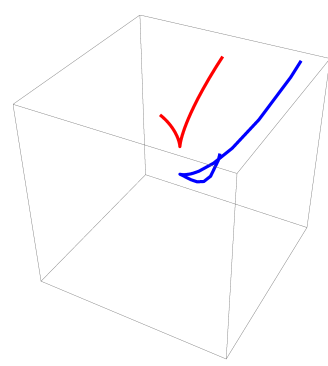

Figure 4. $\gamma$ and $\mathcal{E} v(\gamma)$

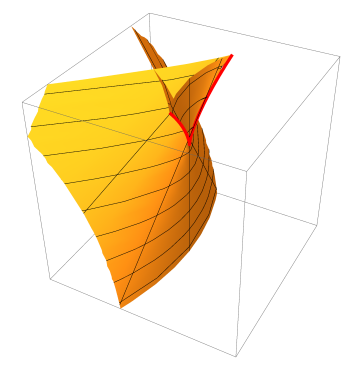

Figure 5. $\mathcal{E} v(\gamma)$ and $\mathcal{F} \mathcal{S}_{\gamma}$

Example 5.2. Let $\left(\gamma, \nu_{1}, \nu_{2}\right):[0,2 \pi) \rightarrow \mathbb{R}^{3} \times \Delta$ be $\gamma(t)=\left(\cos ^{3} t, \sin ^{3} t, \cos 2 t\right)$, $\nu_{1}(t)=(-\sin t,-\cos t, 0), \nu_{2}(t)=(-4 \cos t / 5,4 \sin t / 5,3 / 5)$. We call $\gamma$ the astroid. By a direct alculation, we have $f(t)=36 / 125 \neq 0$. The evolute and focal surface are given by $\mathcal{E} v(\gamma)(t)=\left(28 \cos ^{3} t / 3,28 \sin ^{3} t / 3,-21 \cos 2 t / 4\right), \mathcal{F} \mathcal{S}(\gamma)(t, \lambda)=$

$$
\left(\cos t\left(\frac{4 \lambda}{5}+\cos ^{2} t+\frac{25}{3} \sin ^{2} t\right), \frac{\sin t}{15}(70-12 \lambda+55 \cos 2 t),-\frac{3}{5} \lambda+\cos 2 t\right) .
$$

The astroid (blue curve), the evolute (red curve) and the focal surface of the astroid see Figures 6 and 7. 


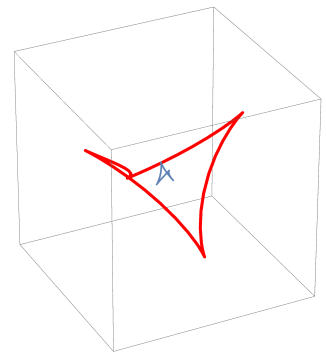

Figure 6. $\gamma$ and $\mathcal{E} v(\gamma)$

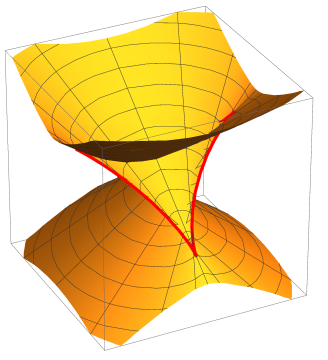

Figure 7. $\mathcal{E} v(\gamma)$ and $\mathcal{F S}(\gamma)$

\section{References}

1 V. I. Arnol'd, Singularities of Caustics and Wave Fronts, Mathematics and its Applications 62, Kluwer Academic Publishers, 1990.

2 R. L. Bishop, There is more than one way to frame a curve, Amer. Math. Monthly 82 (1975) 246-251.

3 J. W. Bruce and P. J. Giblin, Curves and Singularities. A geometrical introduction to singularity theory. Second edition, Cambridge University Press, Cambridge, 1992.

4 D. Fuchs, Evolutes and involutes of spatial curves, Amer. Math. Monthly 120 (2013) $217-231$.

5 T. Fukunaga and M. Takahashi, Existence and uniqueness for Legendre curves, J. Geom. 104 (2013) 297-307.

6 T. Fukunaga and M. Takahashi, Evolutes of fronts in the Euclidean plane. J. Singul. 10 (2014) 92-107.

7 T. Fukunaga and M. Takahashi, Existence conditions of framed curves for smooth curves. J. Geom,. 108 (2017) 763-774.

8 A. Gray, E. Abbena and S. Salamon, Modern Differential Geometry of Curves and Surfaces with Mathematica. Third edition, Studies in Advanced Mathematics. Chapman and Hall/CRC, Boca Raton, FL, 2006.

9 S. Honda, Rectifying developable surfaces of framed base curves and framed helices. to appear in Advanced Studies in Pure Mathematics (2017).

10 S. Honda and M. Takahashi, Framed curves in the Euclidean space. Adv. Geom. 16 (2016) 265-276.

11 S. Honda and M. Takahashi, Evolutes of framed immersions in the Euclidean space. Hokkaido Univ. Preprint Series in Math. 1095,

URL:http://eprints3.math.sci.hokudai.ac.jp/2400/1/pre1095.pdf.

12 S. Izumiya and S. Otani, Flat approximations of surfaces along curves. Demonst. Math. 48 (2015) 217-241.

13 M. Kokubu, W. Rossman, K. Saji, M. Umehara and K. Yamada, Singularities of flat fronts in hyperbolic space, Pacific J. Math. 221 (2005) 303-351.

14 I. R. Porteous, Geometric Differentiation for the Intelligence of Curves and Surfaces. Second edition, Cambridge University Press, Cambridge, 2001.

15 M. C. Romero-Fuster and E. Sanabria-Codesal, Generalized evolutes, vertices and conformal invariants of curves in $\mathbb{R}^{n+1}$, Indag. Math. N. S. 10 (1999) 297-305.

16 R. Uribe-Vargas, On Vertices, focal curvatures and differential geometry of space curves, Bull. Braz. Math. Soc. (N.S.) 36 (2005) 285-307. 